\title{
Integral-equation approach to the calculation of the potential distribution in a fluid
}

\author{
F. Lado \\ Department of Physics, North Carolina State University, Raleigh, North Carolina 27695-8202
}

(Received 9 August 1990)

\begin{abstract}
If a test particle in a fluid is subject to a scalar field from each molecule of the fluid, the total field experienced by the test particle from $N$ fluid molecules is a random variable whose probability density is often denoted a potential distribution. We develop a general procedure for the calculation of the potential distribution at a real test particle (a molecule of the fluid), based on a generalized, complex pair distribution function. The procedure involves the generalization of integral-equation theory of classical fluids to encompass a system with a complex interaction potential. The meanspherical approximation for the same problem is studied to motivate a generalized closure of the integral-equation formalism. With this single approximate ingredient, a closed, coupled pair of nonlinear integral equations is obtained and their numerical solution is outlined. For the Gaussian approximation, a simplified version of the same procedure can be used to compute the second moment of the distribution without invoking the Kirkwood superposition approximation. The general method is applied to the calculation of the potential distribution in a one-component plasma.
\end{abstract}

\section{INTRODUCTION}

A test particle (labeled 0 and located at $\mathbf{r}_{0}$ in a volume $V)$ immersed in a fluid of $N$ molecules filling $V$ experiences a scalar field $\psi\left(r_{0 j}\right)$ due to molecule $j$ at $\mathbf{r}_{j}$. The total field strength

$$
\Psi=\sum_{j=1}^{N} \psi\left(r_{0 j}\right), \quad r_{0 j}=\left|\mathbf{r}_{j}-\mathbf{r}_{0}\right|,
$$

experienced by the test particle due to the $N$ fluid molecules in equilibrium is a random variable of fluid configuration whose probability density is generally called a potential distribution. ${ }^{1-5}$ On the one hand, if the test particle is itself a member of the fluid and so influences the configuration of its environment, we shall say that the test particle is real and define the potential distribution as

$$
P_{R}(\Psi)=\left\langle\delta\left[\Psi-\sum_{j=1}^{N} \psi\left(r_{0 j}\right)\right]\right\rangle_{N+1},
$$

where the angular brackets and subscript denote a thermal average over configurations of the $(N+1)$ molecule system,

$$
\langle F\rangle_{N+1} \equiv \frac{1}{Z_{N+1}} \int d \mathbf{r}_{0} d \mathbf{r}_{1} \cdots d \mathbf{r}_{N} e^{-\beta U_{N+1}} F
$$

with

$$
\begin{aligned}
U_{N+1} & =U\left(\mathbf{r}_{0}, \mathbf{r}_{1}, \ldots, \mathbf{r}_{N}\right) \\
& =\sum_{0 \leq i<j \leq N} \phi\left(r_{i j}\right)
\end{aligned}
$$

and

$$
Z_{N+1}=\int d \mathbf{r}_{0} d \mathbf{r}_{1} \cdots d \mathbf{r}_{N} e^{-\beta U_{N+1}} .
$$

Here, $\phi(r)$ is the intermolecular potential of the fluid and $\beta=(k T)^{-1}$, where $T$ is the absolute temperature. In this paper, we shall be primarily concerned with developing a general procedure for calculating $P_{R}(\Psi)$.

On the other hand, if the test particle does not interact with the fluid molecules, so that its environment is unaffected by its presence, we shall say that the test particle is virtual and define the potential distribution as

$$
P_{V}(\Psi)=\left\langle\delta\left(\Psi-\sum_{J=1}^{N} \psi\left(r_{0 j}\right)\right)\right\rangle_{N},
$$

where correspondingly

$$
\langle F\rangle_{N} \equiv \frac{1}{Z_{N}} \int d \mathbf{r}_{1} \cdots d \mathbf{r}_{N} e^{-\beta U_{N}} F
$$

with

$$
\begin{aligned}
U_{N} & =U\left(\mathbf{r}_{1}, \ldots, \mathbf{r}_{N}\right) \\
& =\sum_{1 \leq i<j \leq N} \phi\left(r_{i j}\right)
\end{aligned}
$$

and

$$
Z_{N}=\int d \mathbf{r}_{1} \cdots d \mathbf{r}_{N} e^{-\beta U_{N}}
$$

In the case where $\psi(r)=\phi(r)$, the intermolecular potential, there is a simple relationship between the two distribution functions. Writing

$$
U_{N+1}=U_{N}+\sum_{j=1}^{N} \phi\left(r_{0 j}\right),
$$

we have 


$$
\begin{aligned}
P_{R}(\Phi) & =\frac{1}{Z_{N+1}} \int d \mathbf{r}_{0} \cdots d \mathbf{r}_{N} e^{-\beta U_{N+1}} \delta\left[\Phi-\sum_{j=1}^{N} \phi\left(r_{0 j}\right)\right] \\
& =\frac{V e^{-\beta \Phi}}{Z_{N+1}} \int d \mathbf{r}_{1} \cdots d \mathbf{r}_{N} e^{-\beta U_{N}} \delta\left[\Phi-\sum_{j-1}^{N} \phi\left(r_{0 j}\right)\right] \\
& =\frac{V Z_{N}}{Z_{N+1}} e^{-\beta \Phi}\left\langle\delta\left[\Phi-\sum_{j=1}^{N} \phi\left(r_{0 j}\right)\right]\right\rangle_{N} \\
& =\frac{V Z_{N}}{Z_{N+1}} e^{-\beta \Phi} P_{V}(\Phi) .
\end{aligned}
$$

But $^{6}$

$$
\frac{V Z_{N}}{Z_{N+1}}=e^{\beta \mu_{\mathrm{ex}}},
$$

where $\mu_{\mathrm{ex}}$ is the excess chemical potential of the fluid, so (1.11) becomes ${ }^{7}$

$$
P_{R}(\Phi)=e^{\beta \mu_{\mathrm{ex}}} e^{-\beta \Phi} P_{V}(\Phi) .
$$

This relation leads directly to some well-known expressions for the chemical potential in terms of potential distributions. Since $P_{R}(\Phi)$ is normalized to unity, we have from (1.13)

$$
1=e^{\beta \mu_{\mathrm{ex}}} \int_{-\infty}^{\infty} d \Phi e^{-\beta \Phi} P_{V}(\Phi)
$$

or

$$
\begin{aligned}
e^{-\beta \mu_{\mathrm{ex}}} & =\int_{-\infty}^{\infty} d \Phi e^{-\beta \Phi} P_{V}(\Phi) \\
& =\left\langle e^{-\beta \Phi}\right\rangle_{N}
\end{aligned}
$$

This result was first obtained by Widom $^{2}$ and Jackson and Klein. ${ }^{3}$

Alternatively, we can rewrite (1.13) as

$$
e^{\beta \mu_{\mathrm{ex}}} P_{V}(\Phi)=e^{\beta \Phi} P_{R}(\Phi)
$$

and obtain, again by normalization,

$$
\begin{aligned}
e^{\beta \mu_{\mathrm{ex}}} & =\int_{-\infty}^{\infty} d \Phi e^{\beta \Phi} P_{R}(\Phi) \\
& =\left\langle e^{\beta \Phi}\right\rangle_{N+1},
\end{aligned}
$$

which is the inverse of $(1.15)^{7,8}$

These expressions for the chemical potential have found application in computer simulations of fluids, ${ }^{7,9-11}$ despite numerical difficulties inherent in the approach.

As noted, we are concerned here primarily with the calculation of $P_{R}(\Psi)$. The basic approach that will be taken is based on an extension of the observation of Mori$\mathrm{ta}^{1}$ regarding the electric (micro)potential and (micro)field distributions in a plasma. (The calculation of the electric microfield distribution, where one seeks to obtain the electric field distribution at ion 0 due to the other charged particles in a plasma, has earlier been reformulated in the same way by Iglesias. ${ }^{12}$ ) This extension is developed in Sec. II and, like the microfield case, leads to a generalized pair distribution function (PDF) for a particle that interacts with other fluid molecules through a complex potential, the calculation of which is described.
Since the mean-spherical approximation ${ }^{13}$ (MSA) proved to be an effective guide in formulating a closure relation for the generalized PDF of the microfield problem, ${ }^{14}$ we study this approximation in Sec. III for the new generalized PDF. The final result there is a Gaussian distribution for $P_{R}(\Psi)$. The Gaussian form is further examined in Sec. IV, with emphasis on the calculation of the needed second moment. The generalized procedure of Sec. II is then used in Sec. $\mathrm{V}$ to obtain the potential distribution in a one-component plasma. Finally, the results of the paper are summarized in Sec. VI.

\section{INTEGRAL-EQUATION APPROACH FOR $P_{R}(\Psi)$}

More accessible to manipulation than $P_{R}(\Psi)$ is its Fourier transform $\widetilde{P}_{R}(K)$, where

$$
\begin{aligned}
P_{R}(\Psi) & =\left\langle\delta\left[\Psi-\sum_{j=1}^{N} \psi\left(r_{0 j}\right)\right]\right\rangle_{N+1} \\
& =\frac{1}{2 \pi} \int_{-\infty}^{\infty} d K e^{i K \Psi}\left\langle e^{-i K \sum_{j=1}^{N} \psi\left(r_{0 j}\right)}\right\rangle_{N+1} \\
& =\frac{1}{2 \pi} \int_{-\infty}^{\infty} d K e^{i K \Psi} \widetilde{P}_{R}(K)
\end{aligned}
$$

so that

$$
\widetilde{P}_{R}(K)=\left\langle e^{-i K \sum_{J}^{N}=1 \psi\left(r_{0 j}\right)}\right\rangle_{N+1} .
$$

As noted some time ago by Morita ${ }^{1}$ for the plasma case, Eq. (2.2) is similar to that for the chemical potential [cf. Eq. (1.17)] and can be handled in a similar manner. Thus differentiation with respect to $K$ gives

$$
\begin{aligned}
\frac{d \ln \widetilde{P}_{R}(K)}{d K} & =-\frac{i}{\widetilde{P}_{R}(K)}\left\langle\sum_{t=1}^{N} \psi\left(r_{0 i}\right) e^{-i K \sum_{J=1}^{N} \psi\left(r_{0 j}\right)}\right\rangle_{N+1} \\
& =-i N \frac{\left\langle\psi\left(r_{01}\right) e^{-i K \sum_{j=1}^{N} \psi\left(r_{0 j}\right)}\right\rangle_{N+1}}{\left\langle e^{-i K \sum_{J=1}^{N} \psi\left(r_{0 j}\right)}\right\rangle_{N+1}} \\
& =-\frac{i N}{V^{2}} \int d \mathbf{r}_{0} d \mathbf{r}_{1} \psi\left(r_{01}\right) g\left(r_{01}, K\right) \\
& =-i \rho \int d \mathbf{r} \psi(r) g(r, K),
\end{aligned}
$$

where $\rho=N / V$. Here,

$$
g\left(r_{01}, K\right)=V^{2} \frac{\int d \mathbf{r}_{2} \cdots d \mathbf{r}_{N} e^{-\beta U_{N+1}} e^{-\imath K \sum_{J=1}^{N} \psi\left(r_{0 j}\right)}}{\int d \mathbf{r}_{0} \cdots d \mathbf{r}_{N} e^{-\beta U_{N+1}} e^{-i K \sum_{J=1}^{N} \psi\left(r_{0 j}\right)}}
$$

is the generalized PDF for a "fluid" interacting through the complex potential

$$
U_{N+1}(K)=U_{N+1}+\frac{i K}{\beta} \sum_{j=1}^{N} \psi\left(r_{0 j}\right) .
$$

That is, we have a two-component system, with particle 0 interacting through $\phi\left(r_{0 j}\right)+i K \psi\left(r_{0 j}\right) / \beta$ with other fluid molecules, which interact among themselves through $\phi(r)$ alone. 
In terms of this generalized PDF, we get $\widetilde{P}_{R}(K)$ in the form

$$
\ln \widetilde{P}_{R}(K)=-i \rho \int_{0}^{K} d \lambda \int d \mathbf{r} \psi(r) g(r, \lambda),
$$

analogous to Iglesias's electric microfield expression. ${ }^{12}$ The task then is to compute $g(r, \lambda)$. [The PDF for the ordinary fluid is $g(r)=g(r, \lambda=0)$. ]

To this end, we apply standard methods of liquid-state theory. ${ }^{6}$ The Ornstein-Zernike (OZ) equations for a twocomponent mixture, with one component at infinite dilution, are

$$
h\left(r_{12}\right)=C\left(r_{12}\right)+\rho \int d \mathbf{r}_{3} h\left(r_{13}\right) C\left(r_{32}\right)
$$

and

$$
h\left(r_{01}, \lambda\right)=C\left(r_{01}, \lambda\right)+\rho \int d \mathbf{r}_{2} h\left(r_{02}, \lambda\right) C\left(r_{21}\right),
$$

where $h=g-1$ and $C(r), C(r, \lambda)$ are, respectively, the direct correlation functions (DCF) of the ordinary liquid with potential $\phi(r)$ and of the solute particle with potential $\phi(r)+i \lambda \psi(r) / \beta$.

Complimenting (2.7) and (2.8) are the closure relations

$$
g(r)=\exp [-\beta \phi(r)+h(r)-C(r)+B(r)]
$$

and

$$
\begin{aligned}
g(r, \lambda)=\exp [ & -\beta \phi(r)-i \lambda \psi(r)+h(r, \lambda)-C(r, \lambda) \\
& +B(r, \lambda)] .
\end{aligned}
$$

Though formally defined by an infinite series in density, the bridge function $B(r)$ in Eq. (2.9) must in practice be approximated, giving rise to the familiar approximate closures in the literature. ${ }^{6}$ We shall assume that an accurate choice for $B(r)$ is available.

The new problems here are first to model the generalized bridge function $B(r, \lambda)$ in Eq. (2.10) and then to solve the resulting complex integral equation for $g(r, \lambda)$. We postpone the question of modeling $B(r, \lambda)$ to Sec. III and consider first how to solve Eqs. (2.8) and (2.10) for given $B(r, \lambda)$.

As with the integral equations of ordinary fluids, it is convenient to introduce the generalized series function

$$
S(r, \lambda)=h(r, \lambda)-C(r, \lambda)
$$

and to deconvolute Eq. (2.8) by Fourier transformation. The result for Eq. (2.8) is

$$
\begin{aligned}
\widetilde{S}(k, \lambda) & =\frac{\rho \widetilde{C}(k) \widetilde{C}(k, \lambda)}{1-\rho \widetilde{C}(k)} \\
& =\rho \widetilde{h}(k) \widetilde{C}(k, \lambda),
\end{aligned}
$$

while for (2.10) we get

$$
\begin{aligned}
C(r, \lambda)= & \exp [-\beta \phi(r)-i \lambda \psi(r)+S(r, \lambda)+B(r, \lambda)] \\
& -1-S(r, \lambda) .
\end{aligned}
$$

Now separate the complex functions into real and imaginary parts,

$$
S(r, \lambda)=S_{1}(r, \lambda)+i S_{2}(r, \lambda),
$$

and similarly for $C(r, \lambda), B(r, \lambda)$, and $h(r, \lambda)$. Equation (2.12) then separates linearly into

$$
\widetilde{S}_{j}(k, \lambda)=\rho \widetilde{h}(k) \widetilde{C}_{j}(k, \lambda), \quad j=1 \text { or } 2,
$$

while from (2.13) we get a coupled pair,

$$
\begin{aligned}
C_{1}(r, \lambda)= & e^{-\beta \phi(r)+S_{1}(r, \lambda)+B_{1}(r, \lambda)} \\
& \times \cos \left[-\lambda \psi(r)+S_{2}(r, \lambda)+B_{2}(r, \lambda)\right] \\
& -1-S_{1}(r, \lambda), \\
C_{2}(r, \lambda)= & e^{-\beta \phi(r)+S_{1}(r, \lambda)+B_{1}(r, \lambda)} \\
& \times \sin \left[-\lambda \psi(r)+S_{2}(r, \lambda)+B_{2}(r, \lambda)\right] \\
& -S_{2}(r, \lambda) .
\end{aligned}
$$

From the definition (2.4) we see that complex conjugation gives

$$
h(r, \lambda)^{*}=h(r,-\lambda),
$$

so that $h_{1}(r, \lambda)$ and $h_{2}(r, \lambda)$ are, respectively, even and odd functions of $\lambda$. The same symmetries carry over to the $\left(C_{1}, C_{2}\right),\left(S_{1}, S_{2}\right)$, and $\left(B_{1}, B_{2}\right)$ pairs.

The solution of Eqs. (2.15)-(2.17) for $S_{1}(r, \lambda)$ and $S_{2}(r, \lambda)$ is by iteration, passing through the Fourier transformations

$$
\begin{aligned}
& \tilde{f}(k)=\frac{4 \pi}{k} \int_{0}^{\infty} d r r f(r) \sin k r, \\
& f(r)=\frac{1}{2 \pi^{2} r} \int_{0}^{\infty} d k k \widetilde{f}(k) \sin k r .
\end{aligned}
$$

Once converged solutions are obtained we compute

$$
\begin{aligned}
g_{1}(r, \lambda)= & e^{-\beta \phi(r)+S_{1}(r, \lambda)+B_{1}(r, \lambda)} \\
& \times \cos \left[-\lambda \psi(r)+S_{2}(r, \lambda)+B_{2}(r, \lambda)\right], \\
g_{2}(r, \lambda)= & e^{-\beta \phi(r)+S_{1}(r, \lambda)+B_{1}(r, \lambda)} \\
& \times \sin \left[-\lambda \psi(r)+S_{2}(r, \lambda)+B_{2}(r, \lambda)\right],
\end{aligned}
$$

and update the integrals for

$$
\begin{aligned}
\ln \widetilde{P}_{R}(K) & =-i \rho \int_{0}^{K} d \lambda \int d \mathbf{r} \psi(r)\left[g_{1}(r, \lambda)+i g_{2}(r, \lambda)\right] \\
& =-F_{1}(K)+i F_{2}(K)
\end{aligned}
$$

where

$$
\begin{aligned}
& F_{1}(K)=\int_{0}^{K} d \lambda u_{2}(\lambda), \\
& F_{2}(K)=\int_{0}^{K} d \lambda u_{1}(\lambda),
\end{aligned}
$$

and

$$
u_{j}(\lambda)=-\rho \int d \mathbf{r} \psi(r) g_{j}(r, \lambda), \quad j=1 \text { or } 2 .
$$

It should be noted that $F_{1}(K)$ and $F_{2}(K)$ are, respectively, even and odd functions of $K$.

Equations $(2.15)-(2.17)$ are to be solved for incrementally increasing $\lambda$ until a "large enough" value is reached. Equation (2.1) is finally evaluated as 
$P_{R}(\Psi)=\frac{1}{\pi} \int_{0}^{\infty} d K e^{-F_{1}(K)} \cos \left[K \Psi+F_{2}(K)\right]$,

so that "large enough" means that the exponential in the integrand of Eq. (2.27) has become negligibly small.

It was remarked at the start of this section that the calculation of $\ln \widetilde{P}_{R}(K)$ parallels that of $\beta \mu_{\mathrm{ex}}$. This analogy can actually be made an equality, as follows. For the case where $\psi(r)=\phi(r)$, we have from (1.17) and (2.1)

$$
\begin{aligned}
e^{\beta \mu_{\mathrm{ex}}} & =\int_{-\infty}^{\infty} d \Phi e^{\beta \Phi} P_{R}(\Phi) \\
& =\frac{1}{2 \pi} \int_{-\infty}^{\infty} d \Phi e^{\beta \Phi} \int_{-\infty}^{\infty} d K e^{i K \Phi} \widetilde{P}_{R}(K) \\
& =\frac{1}{2 \pi} \int_{-\infty}^{\infty} d K \widetilde{P}_{R}(K) \int_{-\infty}^{\infty} d \Phi e^{i(K-i \beta) \Phi} \\
& =\int_{-\infty}^{\infty} d K \widetilde{P}_{R}(K) \delta(K-i \beta)=\widetilde{P}_{R}(i \beta)
\end{aligned}
$$

so that, with (2.6),

$$
\begin{aligned}
\beta \mu_{\mathrm{ex}} & =\ln \widetilde{P}_{R}(i \beta) \\
& =-i \rho \int_{0}^{i \beta} d \lambda \int d \mathbf{r} \phi(r) g(r, \lambda) \\
& =\rho \int_{0}^{1} d \xi \int d \mathbf{r} \beta \phi(r) g(r, i \beta(1-\xi))
\end{aligned}
$$

But from Eq. (2.4) with $\psi(r)=\phi(r)$,

$g\left[r_{01}, i \beta(1-\xi)\right]=V^{2} \frac{\int d \mathbf{r}_{2} \cdots d \mathbf{r}_{N} e^{-\beta U_{N}} e^{-\xi \beta \sum_{j=1}^{N} \phi\left(r_{0 j}\right)}}{\int d \mathbf{r}_{0} \cdots d \mathbf{r}_{N} e^{-\beta U_{N}} e^{-\xi \beta \sum_{j=1}^{N} \phi\left(r_{0 j}\right)}}$

is just the PDF of an $(N+1)$-molecule system in which the presence of molecule 0 is "turned on" to the extent $\xi$, $0 \leq \xi \leq 1$, making Eq. (2.29), despite the awkward notation, the usual recipe for the excess chemical potential. ${ }^{15}$

\section{MSA FOR THE POTENTIAL DISTRIBUTION PROBLEM}

Essentially nothing is known about the generalized bridge function $B(r, \lambda)$ needed in Eq. (2.10). For the similar problem of the electric microfield distribution, the MSA turned out to provide a very successful ansatz for the generalized bridge function. ${ }^{14}$ Thus we are encouraged to consider its results in the present calculation.

To set up the MSA, one must first insert a hard core of diameter $\sigma$ into every particle of the fluid. Then the $\mathrm{OZ}$ equation (2.8),

$$
\tilde{h}(k, \lambda)=\frac{\widetilde{C}(k, \lambda)}{1-\rho \widetilde{C}(k)},
$$

is combined with the exact hard-core condition

$$
h(r, \lambda)=-1, \quad r<\sigma
$$

and the approximation (MSA)

$$
C(r, \lambda)=-\beta \phi(r)-i \lambda \psi(r), \quad r>\sigma
$$

to yield a closed system of equations for $h(r, \lambda)$ and $C(r, \lambda)$.

Separating out as before the real and imaginary parts, Eqs. (3.2) and (3.3) become

$$
\begin{aligned}
& h_{1}(r, \lambda)=-1, \quad r<\sigma \\
& C_{1}(r, \lambda)=-\beta \phi(r), \quad r>\sigma
\end{aligned}
$$

and

$$
\begin{aligned}
& h_{2}(r, \lambda)=0, \quad r<\sigma \\
& C_{2}(r, \lambda)=-\lambda \psi(r), \quad r>\sigma .
\end{aligned}
$$

These are to be joined with

$$
\tilde{h}_{j}(k, \lambda)=\frac{\widetilde{C}_{j}(k, \lambda)}{1-\rho \widetilde{C}(k)}, j=1 \text { or } 2 .
$$

Equations (3.4) and (3.5), with (3.8), are just the ordinary MSA equations for a fluid with intermolecular potential $\phi(r)$ outside the hard core, so that $\lambda$ plays no role and

$$
\begin{aligned}
& h_{1}(r, \lambda)=h(r), \\
& C_{1}(r, \lambda)=C(r) .
\end{aligned}
$$

The situation is not so obvious with regard to the imaginary parts, since Eq. (3.6) lacks the needed core condition.

Consider, however, the linear combinations

$$
h_{ \pm}(r, \lambda)=h_{1}(r, \lambda) \pm \frac{\beta}{\lambda} h_{2}(r, \lambda)
$$

and similarly for $C_{ \pm}(r, \lambda)$. Then evidently

$$
h_{ \pm}(r, \lambda)=-1, \quad r<\sigma
$$




$$
C_{ \pm}(r, \lambda)=-\beta[\phi(r) \pm \psi(r)], \quad r>\sigma
$$

while the Fourier transforms satisfy

$$
\widetilde{h}_{ \pm}(k, \lambda)=\frac{\widetilde{C}_{ \pm}(k, \lambda)}{1-\rho \widetilde{C}(k)} .
$$

The parameter $\lambda$ has now again vanished and the solutions are manifestly

$$
h_{ \pm}(r, \lambda)=h(r \mid \phi \pm \psi)
$$

and similarly for $C_{ \pm}(r, \lambda)$. Here $h(r \mid \chi), C(r \mid \chi)$ are the solutions to

$$
\widetilde{h}(k \mid \chi)=\frac{\widetilde{C}(k \mid \chi)}{1-\rho \widetilde{C}(k)},
$$

with

$$
\begin{aligned}
& h(r \mid \chi)=-1, \quad r<\sigma \\
& C(r \mid \chi)=-\beta \chi(r), \quad r>\sigma .
\end{aligned}
$$

Because the denominator in (3.16) retains the DCF for the potential $\phi(r)$, these are not the usual MSA equations, unless $\chi=\phi$. However, the problem is well posed and we need only the form of the solutions.

Then combining these results we have finally

$$
\begin{aligned}
h_{1}(r, \lambda) & =\frac{1}{2}[h(r \mid \phi+\psi)+h(r \mid \phi-\psi)] \\
& =h(r) \equiv h(r \mid \phi), \\
h_{2}(r, \lambda) & =\frac{\lambda}{2 \beta}[h(r \mid \phi+\psi)-h(r \mid \phi-\psi)],
\end{aligned}
$$

and similar equations for $C_{1}(r, \lambda)$ and $C_{2}(r, \lambda)$. Note that either $h(r \mid \phi+\psi)$ or $h(r \mid \phi-\psi)$ could be eliminated from (3.20) in favor of $h(r)$ using (3.19), giving variants of Eq. (3.20) that underscore the linear dependence of the MSA $h(r \mid \chi)$ on $\chi$. In fact, we may conclude from this linearity that

$$
\begin{aligned}
\frac{d}{d \xi} h(r \mid \phi+\xi \psi) & \equiv \dot{h}(r \mid \phi+\zeta \psi) \\
& =\dot{h}(r \mid \phi)
\end{aligned}
$$

does not depend on $\zeta$, so that

$$
\begin{aligned}
\frac{1}{2}[h(r \mid \phi+\psi)-h(r \mid \phi-\psi)] & =\frac{1}{2} \int_{-1}^{1} d \zeta \frac{d}{d \zeta} h(r \mid \phi+\zeta \psi) \\
& =\dot{h}(r \mid \phi)
\end{aligned}
$$

which finally gives

$$
\begin{aligned}
& h_{1}(r, \lambda)=h(r \mid \phi), \\
& h_{2}(r, \lambda)=\frac{\lambda}{\beta} \dot{h}(r \mid \phi),
\end{aligned}
$$

and similarly for $C_{1}(r, \lambda)$ and $C_{2}(r, \lambda)$.

The calculation of the potential distribution then proceeds with

$$
\begin{aligned}
& u_{1}(\lambda)=-\rho \int d \mathbf{r} \psi(r) g(r \mid \phi)=-c_{1}, \\
& u_{2}(\lambda)=-\frac{\lambda}{\beta} \rho \int d \mathbf{r} \psi(r) \dot{g}(r \mid \phi)=\lambda c_{2},
\end{aligned}
$$

so that

$$
\begin{aligned}
& F_{1}(K)=\frac{1}{2} c_{2} K^{2}, \\
& F_{2}(K)=-c_{1} K,
\end{aligned}
$$

and

$$
\widetilde{P}_{R}(K)=\exp \left(-i c_{1} K-\frac{1}{2} c_{2} K^{2}\right),
$$

where we have used general expressions from Sec. II. Thus, in the mean-spherical approximation, we find that the potential distribution is Gaussian,

$$
\begin{aligned}
P_{R}(\Psi) & =\frac{1}{2 \pi} \int_{-\infty}^{\infty} d K e^{-(1 / 2) c_{2} K^{2}} e^{i\left(\Psi-c_{1}\right) K} \\
& =\frac{1}{\left(2 \pi c_{2}\right)^{1 / 2}} e^{-\left(\Psi-c_{1}\right)^{2} / 2 c_{2}},
\end{aligned}
$$

with mean $c_{1}$ and variance $c_{2}$. It will be shown in Sec. IV that Eqs. (3.25) and (3.26) are exact expressions for the first moment $c_{1}$ and second central moment $c_{2}$ of the potential distribution $P_{R}(\Psi)$.

We take away from these results the suggestion that the needed ansatz for $B(r, \lambda)$ is

$$
B(r, \lambda)=B(r \mid \phi)+i \frac{\lambda}{\beta} \dot{B}(r \mid \phi),
$$

where

$$
\left.\dot{B}(r \mid \phi) \equiv \frac{d}{d \zeta} B(r \mid \phi+\xi \psi)\right|_{\zeta=0}
$$

and $B(r \mid \chi)$ is the bridge function of a solute particle with potential $\chi(r)$ in a fluid with intermolecular potential $\phi(r)$, without any restriction now to the MSA.

Equation (3.31) is the only approximation in the calculation.

\section{GAUSSIAN SHAPE FOR $P_{R}(\Psi)$}

Section III showed that use of the MSA in computing $g(r, \lambda)$ leads to a Gaussian form for $P_{R}(\Psi)$, Eq. (3.30). It is not to be expected that a Gaussian will always be a good approximation to $P_{R}(\Psi)$ (see, e.g., Ref. 5) and the general approach of Sec. II is in fact not constrained to such an outcome. Nevertheless, a Gaussian shape often does serve well and it is worth examining here some further details. The calculation of the second moment is a special concern.

Moments of $P_{R}(\Psi)$ are defined by the expansion of the characteristic function

$$
\begin{aligned}
\widetilde{P}_{R} & =\int_{-\infty}^{\infty} d \Psi e^{-i K \Psi} P_{R}(\Psi) \\
& =\left\langle e^{-i K \Sigma_{j=1}^{N} \psi\left(r_{0 j}\right)}\right\rangle \\
& =1-i m_{1} K-\frac{1}{2 !} m_{2} K^{2}+\frac{i}{3 !} m_{3} K^{3}+\cdots,
\end{aligned}
$$

where

$$
m_{n}=\int_{-\infty}^{\infty} d \Psi \Psi^{n} P_{R}(\Psi)
$$


or

$$
m_{n}=\left\langle\left(\sum_{j=1}^{N} \psi\left(r_{0 j}\right)\right)^{n}\right\rangle_{N+1} .
$$

The more useful cumulant expansion obtained from this is

$$
\ln \widetilde{P}_{R}(K)=-i c_{1} K-\frac{1}{2 !} c_{2} K^{2}+\frac{i}{3 !} c_{3} K^{3}+\cdots,
$$

where

$$
\begin{aligned}
& c_{1}=m_{1}, \\
& c_{2}=m_{2}-m_{1}^{2}, \\
& c_{3}=m_{3}-3 m_{2} m_{1}+2 m_{1}^{3},
\end{aligned}
$$

:

The first moment is easily evaluated from (4.3) as

$$
c_{1}=m_{1}=\rho \int d \mathbf{r} \psi(r) g(r),
$$

where $g(r)$ is again the PDF of the ordinary fluid with intermolecular potential $\phi(r)$. It will be noted that Eq. (4.6) is the same as the MSA result for $c_{1}$, Eq. (3.25).

Similarly, the second moment from Eq. (4.3) is obtained as

$$
\begin{aligned}
m_{2}= & N\left\langle\psi^{2}\left(r_{01}\right)\right\rangle_{N+1}+N(N-1)\left\langle\psi\left(r_{01}\right) \psi\left(r_{02}\right)\right\rangle_{N+1} \\
= & \rho \int d \mathbf{r} \psi^{2}(r) g(r) \\
& +\rho^{2} \int d \mathbf{r}_{01} d \mathbf{r}_{02} \psi\left(r_{01}\right) \psi\left(r_{02}\right) g^{(3)}\left(\mathbf{r}_{01}, \mathbf{r}_{02}\right)
\end{aligned}
$$

and thus

$$
\begin{aligned}
& c_{2}=\rho \int d \mathbf{r} \psi^{2}(r) g(r) \\
&+\rho^{2} \int d \mathbf{r}_{01} d \mathbf{r}_{02} \psi\left(r_{01}\right) \psi\left(r_{02}\right) \\
& \quad \times\left[g^{(3)}\left(\mathbf{r}_{01}, \mathbf{r}_{02}\right)-g\left(r_{01}\right) g\left(r_{02}\right)\right] .
\end{aligned}
$$

Equation (4.7) brings in the three-particle correlation function ${ }^{6} g^{(3)}$ for the fluid with intermolecular potential $\phi(r)$. This is generally a little-known quantity and the usual expedient for such situations is to use the Kirkwood superposition approximation,

$$
g^{(3)}\left(\mathbf{r}_{01}, \mathbf{r}_{02}\right) \approx g\left(r_{01}\right) g\left(r_{02}\right) g\left(r_{12}\right) .
$$

With this, Eq. (4.8) becomes $^{5}$

$$
\begin{aligned}
c_{2} \approx & \rho \int d \mathbf{r} w(r) \psi(r) \\
& +\rho^{2} \int d \mathbf{r}_{1} d \mathbf{r}_{2} w\left(r_{01}\right) h\left(r_{12}\right) w\left(r_{20}\right),
\end{aligned}
$$

where

$$
w(r)=\psi(r) g(r) .
$$

The convolution integral of (4.10) is now readily evaluated using Fourier transforms. The cost is an unknown effect on the accuracy of $c_{2}$ from using (4.9).

Alternatively, differentiation of Eq. (2.4) with respect to $K$ gives

$$
\left.\frac{d}{d K} g(r, K)\right|_{K=0} \equiv g^{\prime}(r, 0)=i g_{2}^{\prime}(r, 0),
$$

with

$$
\begin{aligned}
g_{2}^{\prime}\left(r_{01}, 0\right)= & -\psi\left(r_{01}\right) g\left(r_{01}\right) \\
& -\rho \int d \mathbf{r}_{2} \psi\left(r_{02}\right)\left[g^{(3)}\left(\mathbf{r}_{01}, \mathbf{r}_{02}\right)\right. \\
& \left.-g\left(r_{01}\right) g\left(r_{02}\right)\right],
\end{aligned}
$$

so that $(4.8)$ can be written

$$
c_{2}=-\rho \int d \mathbf{r} \psi(r) g_{2}^{\prime}(r, 0) .
$$

Noting from the definitions of dot and prime operations, Eqs. (3.32) and (4.12), that

$$
-i g^{\prime}(r, 0)=g_{2}^{\prime}(r, 0)=\frac{1}{\beta} \dot{g}(r \mid \phi),
$$

we see that the MSA expression (3.26) for $c_{2}$ also matches the exact result above.

The quantity $g_{2}^{\prime}(r, 0)$ in $(4.14)$ can be computed by the method of Sec. II. From (2.22), we get that

$$
\begin{aligned}
g_{2}^{\prime}(r, 0) & =h_{2}^{\prime}(r, 0) \\
& =g(r)\left[-\psi(r)+S_{2}^{\prime}(r, 0)+B_{2}^{\prime}(r, 0)\right] \\
& =C_{2}^{\prime}(r, 0)+S_{2}^{\prime}(r, 0),
\end{aligned}
$$

while from (2.12)

$$
\widetilde{S}_{2}^{\prime}(k, 0)=\rho \widetilde{h}(k) \widetilde{C}_{2}^{\prime}(k, 0) .
$$

The iterative solution of these two equations yields the $g_{2}^{\prime}(r, 0)$ to be used in (4.14). The function $B_{2}^{\prime}(r, 0)$ in (4.16) is obtained from Eq. (3.31).

Note that when (4.16) is inserted into Eq. (4.14) we find

$$
\begin{aligned}
c_{2}= & \rho \int d \mathbf{r} \psi^{2}(r) g(r) \\
& -\rho \int d \mathbf{r} \psi(r) g(r)\left[S_{2}^{\prime}(r, 0)+B_{2}^{\prime}(r, 0)\right] .
\end{aligned}
$$

Comparison with (4.8) shows the replacement for $g^{(3)}$ effected by this method.

A third way of computing $c_{2}$ is to first obtain $P_{R}(\Psi)$ by the general method of Sec. II and then evaluate the first and second moments directly from (4.2). This procedure should yield essentially the same result as Eq. (4.14) and can serve as an internal check in a calculation. In addition, comparison of the general solution with the Gaussian approximation can test the reasonableness of the latter.

If, for the case $\psi(r)=\phi(r)$, the Gaussian approximation is indeed reasonably accurate, then the chemical potential and the potential distribution $P_{V}(\Phi)$ at a virtual particle can also be expressed in terms of $c_{1}$ and $c_{2}$. From Eq. (1.17), we get in this case

$$
\begin{aligned}
\mu_{\mathrm{ex}} & =\frac{1}{\beta} \ln \left(\frac{1}{\left(2 \pi c_{2}\right)^{1 / 2}} \int_{-\infty}^{\infty} d \Phi e^{\beta \Phi} e^{-\left(\Phi-c_{1}\right)^{2} / 2 c_{2}}\right) \\
& =c_{1}+\frac{1}{2} \beta c_{2},
\end{aligned}
$$


which, with Eq. (1.13), yields another Gaussian for the potential distribution at a virtual particle,

$$
P_{V}(\Phi)=\frac{1}{\left(2 \pi c_{2}\right)^{1 / 2}} e^{-\left(\Phi-c_{1}-\beta c_{2}\right)^{2} / 2 c_{2}}
$$

\section{V. $P_{R}(\Phi)$ IN THE ONE-COMPONENT PLASMA}

The potential distribution in a one-component plasma (OCP) has been obtained only in the Debye-Hückel (DH) limit, by Morita ${ }^{1}$ and later by Jackson and Klein. ${ }^{3}$ The result for $P_{R}(\Phi)$ in this case is a Gaussian,

$$
P_{R}(\Phi)=\frac{1}{\left(2 \pi c_{2}\right)^{1 / 2}} e^{-\left(\Phi-c_{1}\right)^{2} / 2 c_{2}},
$$

with

$$
\begin{aligned}
& c_{1}=-e^{2}\left(4 \pi \rho \beta e^{2}\right)^{1 / 2}, \\
& c_{2}=-c_{1} / \beta .
\end{aligned}
$$

For these values, Eq. (4.19) yields

$$
\mu_{\mathrm{ex}}=c_{1} / 2 \text {. }
$$

In this section, we report solutions of the generalized integral equation formulation for $P_{R}(\Phi)$ in a OCP. For this case, a few special modifications of the previous formalism must be made.

The OCP model consists of discrete point changes $e$ in a uniform neutralizing background; i.e., actually two components but only one with configurational structure. The effective potential between the point charges is then

$$
\phi_{\mathrm{OCP}}(r)=\phi(r)-\frac{1}{V} \int d \mathbf{R} \phi(R)
$$

where $\phi(r)=e^{2} / r$ is the Coulomb potential and the integral term in (5.5) arises from the uniform background.

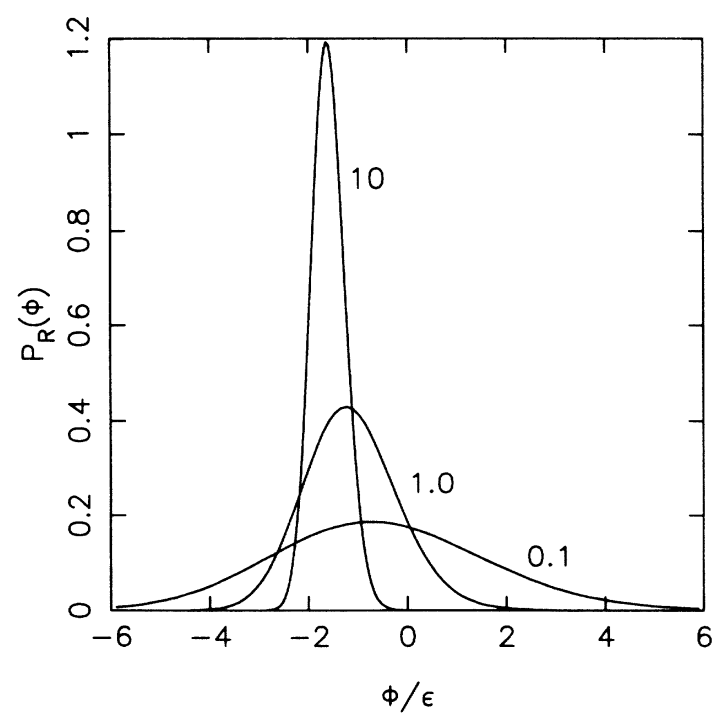

FIG. 1. Potential distribution at a real particle in a OCP at three values of $\Gamma=\beta e^{2} / a$.
TABLE I. Excess chemical potential in the OCP, $\mu_{\mathrm{ex}} / \varepsilon$.

\begin{tabular}{rrrr}
\hline$\Gamma$ & Eq. (5.4) & Eq. (4.19) & RHNC \\
\hline 0.1 & -0.264 & -0.269 & -0.262 \\
1.0 & -0.866 & -0.687 & -0.627 \\
10.0 & -2.739 & -1.028 & -0.976 \\
\hline
\end{tabular}

This has the effect of replacing the PDF $g$ by $h=g-1$ in certain expressions, specifically the basic Eq. (2.6) for $\widetilde{P}_{R}(K)$ and Eq. (4.6) for the first moment $m_{1}$. The second moment $m_{2}$ also picks up additional terms but Eq. (4.8) for $c_{2}$ remains unchanged. [The function $\psi(r)$ in these expressions is replaced by the Coulomb potential $\phi(r)$.] Additionally, the long-range nature of the Coulomb potential requires special numerical handling. In solving the equations of Sec. II for $g(r, \lambda)$, we have adopted the separation of $\phi(r)$ into long- and short-range parts used by $\mathrm{Ng},{ }^{16}$ along with the corresponding separation of the generalized functions $C(r, \lambda)$ and $S(r, \lambda)$. The long-range parts are then dealt with analytically.

Finally, the ordinary PDF $g(r)$ for the OCP needed in the calculation was obtained using the optimized reference hypernetted-chain (RHNC) equation, as described elsewhere. ${ }^{17,18}$ In this scheme, the bridge function of a hard-sphere fluid, $B_{\mathrm{HS}}(r ; \sigma)$, is used to model the corresponding function of the OCP, as suggested originally by Rosenfeld and Ashcroft. ${ }^{19}$ This same quantity is also used here for the real part of the generalized function $B(r, \lambda)$, Eq. (3.31). In the same spirit, since the hardsphere diameter $\sigma$ is the variable parameter of this potential, we put

$$
\dot{B}(r \mid \phi)=\sigma \frac{d B_{\mathrm{HS}}(r ; \sigma)}{d \sigma}\left[\frac{d \ln \sigma(\xi)}{d \xi}\right]_{\xi=0}
$$

for the imaginary part, Eq. (3.32). The derivative of $\sigma(\zeta)$ is obtained numerically by solving the optimized RHNC equation for the potential $\phi(r)+\zeta \psi(r)$ on the solute particle. As usual, distances in the calculation are scaled by the ion-sphere radius $a=(3 / 4 \pi \rho)^{1 / 3}$ and energies by $\varepsilon=e^{2} / a$.

The results of the calculation of $P_{R}(\Phi)$ are shown in Fig. 1 for values of $\Gamma=\beta e^{2} / a=0.1,1.0$, and 10 . In all the cases, the shapes are very close to but not quite Gaussian. (On the scale of the figure the differences would not be clearly distinguishable.) One measure of the discrepancy can be found in Table I, where values of $\mu_{\mathrm{ex}}$ given by the Gaussian formula based on the first two moments, Eq. (4.19), are compared with the direct RHNC results. ${ }^{18}$ For further comparison, the $\mathrm{DH}$ values, Eq.

TABLE II. Mean of the potential distribution in the OCP, $\underline{c_{1} / \varepsilon .}$

\begin{tabular}{rrrr}
\hline \hline$\Gamma$ & Eq. (5.2) & Eq. (4.6) & Eq. (4.2) \\
\hline 0.1 & -0.548 & -0.514 & -0.514 \\
1.0 & -1.732 & -1.141 & -1.141 \\
10.0 & -5.477 & -1.598 & -1.598 \\
\hline \hline
\end{tabular}


TABLE III. Variance of the potential distribution in the $\mathrm{OCP}, c_{2} / \varepsilon^{2}$.

\begin{tabular}{rcccc}
\hline \hline$\Gamma$ & Eq. (5.3) & Eq. (4.10) & Eq. (4.14) & Eq. (4.2) \\
\hline 0.1 & 5.477 & 4.981 & 4.897 & 4.886 \\
1.0 & 1.732 & 1.277 & 0.909 & 0.906 \\
10.0 & 0.548 & 0.361 & 0.114 & 0.113 \\
\hline \hline
\end{tabular}

(5.4), are also included in the table and serve as a check in the small- $\Gamma$ limit.

The computed mean $c_{1}$ and variance $c_{2}$ of the distributions are listed in Tables II and III, respectively. The general trend of deepening mean and narrowing variance is of course present in the DH formulas, but the quantitative agreement of these with the more general calculation presented here rapidly decreases with increasing $\Gamma$, as expected. Values of $c_{1}$ in Table II obtained from the ordinary PDF through Eq. (4.6) are seen to be in excellent agreement with the mean values found directly from the distribution $P_{R}(\Phi)$ through Eq. (4.2). These two evaluations of $c_{1}$ are entirely independent.

Table III offers two comparisons to the variance obtained directly from $P_{R}(\Phi)$ through Eq. (4.2), in addition to the DH values for completeness. The data in the third column are based on the use of the superposition approximation, Eq. (4.10), while those in the fourth column are from Eq. (4.14). As expected, the latter agree very well with the direct values in the last column; they are after all based on similar procedures. Use of the superposition approximation, however, appears to lead to significant error, except for very small $\Gamma$, for which, as the low-density limit, the superposition approximation is valid. These results suggest that Eq. (4.10) for $c_{2}$ should be used with caution. [We remark that the "Gaussian" $\mu_{\mathrm{ex}}$ values in Table I were obtained using $c_{2}$ from Eq. (4.14).] The coefficient of skewness, $c_{3} /\left(c_{2}\right)^{3 / 2}$, which vanishes for Gaussians, is computed to be $0.33,0.29$, and 0.18 for $\Gamma=0.1,1.0$, and 10 , respectively.

\section{CONCLUSIONS}

Potential distribution theory has been employed for some time as an alternative path to the calculation of the chemical potential in computer simulations. Statistical scatter can be a serious problem in this approach, but re- sults for $P_{R}(\Phi)$ have been reported for the Lennard-Jones fluid. ${ }^{7,11,20}$ Analytic or semianalytic calculations, however, appear to be almost entirely restricted to the Gaussian approximation (including the Debye-Hückel limit in plasmas ${ }^{1,3}$ ) or the limit of no molecular correlations. (See, however, Ref. 5.) In the latter limit, the generalized PDF is simply

$$
g(r, \lambda)=e^{-\imath \lambda \psi(r)},
$$

which leads to the Holtsmark approximation, ${ }^{21}$

$$
\widetilde{P}(K)=\exp \left[\rho \int d \mathbf{r}\left(e^{-i K \psi(r)}-1\right)\right] .
$$

(There is obviously no difference here between real and virtual test particles.)

In this paper, we have outlined a general procedure that will allow the calculation of the potential distribution $P_{R}(\Psi)$ at a real test particle for arbitrarily strong interparticle correlations and arbitrary potential. For distributions that are approximately Gaussian, the same procedure provides an alternative route to the needed second moment that avoids invoking the uncontrolled Kirkwood superposition approximation.

This general method is based on integral equation theory for classical fluids and requires specification of a so-called generalized bridge function $B(r, \lambda)$ to close these equations. Earlier calculations of the microfield distribution in a plasma ${ }^{14}$ suggest that the mean-spherical approximation can provide guidance on this closure. We have therefore examined the MSA for this problem and find that $P_{R}(\Psi)$ in this approximation is always Gaussian with exact functional forms for the first two moments. For the broader question of $B(r, \lambda)$, we have extracted the ansatz

$$
B(r, \lambda)=B(r \mid \phi)+i \frac{\lambda}{\beta}\left[\left.\frac{d}{d \zeta} B(r \mid \phi+\zeta \psi)\right|_{\zeta=0},\right.
$$

where $B(r \mid \chi)$ is the bridge function of a solute particle with potential $\chi(r)$ in a fluid with intermolecular potential $\phi(r)$. Equation (6.3) is the only approximation in the procedure.

The general scheme has been illustrated with an application to the OCP. Internal consistency checks based on computed moments indicate good accuracy for the method.
${ }^{1}$ T. Morita, Prog. Theor. Phys. 23, 1211 (1960).

${ }^{2}$ B. Widom, J. Chem. Phys. 39, 2808 (1963).

${ }^{3}$ J. L. Jackson and L. S. Klein, Phys. Fluids 7, 228 (1964).

${ }^{4}$ B. Widom, J. Phys. Chem. 86, 869 (1982).

${ }^{5}$ S. H. Simon, V. Dobrosavljević, and R. M. Stratt, J. Chem. Phys. 93, 2640 (1990).

${ }^{6}$ See, e.g., J. P. Hansen and I. R. McDonald, Theory of Simple Liquids (Academic, London, 1986).

${ }^{7}$ K. S. Shing and K. E. Gubbins, Mol. Phys. 46, 1109 (1982).

${ }^{8}$ M. J. de Oliveira, cited by Widom in Ref. 4.

${ }^{9}$ D. J. Adams, Mol. Phys. 28, 1241 (1974).

${ }^{10}$ S. Romano and K. Singer, Mol. Phys. 37, 1765 (1979).

${ }^{11}$ J. G. Powles, W. A. B. Evans, and N. Quirke, Mol. Phys. 46, 1347 (1982).
${ }^{12}$ C. A. Iglesias, Phys. Rev. A 27, 2705 (1983).

${ }^{13}$ J. L. Lebowitz and J. K. Percus, Phys. Rev. 144, 251 (1966).

${ }^{14}$ F. Lado, Phys. Rev. A 34, 4131 (1986); 36, 313 (1987).

${ }^{15}$ See, e.g., D. A. McQuarrie, Statistical Mechanics (Harper and Row, New York, 1976), p. 264.

${ }^{16}$ K.-C. Ng, J. Chem. Phys. 61, 2680 (1974).

${ }^{17}$ F. Lado, Phys. Lett. 89A, 196 (1982).

${ }^{18}$ F. Lado, S. M. Foiles, and N. W. Ashcroft, Phys. Rev. A 28, 2374 (1983).

${ }^{19}$ Y. Rosenfeld and N. W. Ashcroft, Phys. Rev. A 20, 1208 (1979).

${ }^{20}$ I. R. McDonald and K. Singer, J. Chem. Phys. 47, 4766 (1967).

${ }^{21}$ J. Holtsmark, Ann. Phys. (Leipzig) 58, 577 (1919). 